\title{
Honneth: Contribuições para o cuidar em enfermagem à luz do amor, direito e solidariedade
}

Honneth: Contributions to Nursing Care in Light of Love, Rights and Solidarity

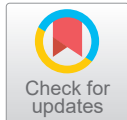

Honneth: Contribuciones para cuidar en enfermería a la luz del amor, la ley y la solidaridad

Como citar este artigo: Jesus, Stefhanie Conceição de; Farias, Carolina Huller; Schneider, Dulcinéia Ghizoni; Schoeller, Soraia Dornelles; Bertoncello, Kátia Cilene Godinho. Honneth: Contribuições para o cuidar em enfermagem à luz do amor, direito e solidariedade. Revista Cuidarte. 2021;12(1):e1201. http://dx.doi.org/10.15649/cuidarte.1201

Revista Cuidarte

doi) Rev Cuid. Ene. - Abril. 2021; 12(1): e1201 http://dx.doi.org/10.15649/cuidarte.1201

E-ISSN: 2346-3414

(1) Stefhanie Conceição de Jesus

(1) Carolina Huller Farias ${ }^{2}$

(1) Dulcinéia Ghizoni Schneider ${ }^{3}$

(1) Soraia Dornelles Schoeller ${ }^{4}$

(1) Kátia Cilene Godinho Bertoncello ${ }^{5}$

1 Enfermeira. Mestranda do Programa de Pós-graduação da Universidade Federal de Santa Catarina. Florianópolis, Santa Catarina, Brasil. E-mail: stefhaniec.j@gmail.com

2 Enfermeira. Mestranda do Programa de Pós-graduação da Universidade Federal de Santa Catarina. Florianópolis, Santa Catarina, Brasil. E-mail: carolinahuller@gmail.com Autor Correspondente

3 Enfermeira. Doutora em Enfermagem. Docente na Universidade Federal de Santa Catarina. Florianópolis, Santa Catarina, Brasil.

E-mail: dulcineia.schneider@ufsc.br

4 Enfermeira. Doutora em Filosofia da Saúde e Enfermagem. Docente na Universidade Federal de Santa Catarina. Florianópolis, Santa Catarina, Brasil.

E-mail: soraia.dornelles@ufsc.br.

5 Enfermeira. Doutora em Enfermagem. Chefe do Departamento de Enfermagem e docente na Universidade Federal de Santa Catarina. Florianópolis, Santa Catarina, Brasil. E-mail: kbertoncello@yahoo.com.br.

\section{Resumo}

Introdução: Compreender a relação intersubjetiva que permeia o cuidado de enfermagem requer reconhecimento recíproco enfermeiro e paciente e/ou familiar. Para tanto, é necessário refletir sobre a construção do próprio ser humano consolidado no amor, no direito e na solidariedade. Objetivo: Refletir sobre o saber e fazer do cuidado de enfermagem a partir do respeito aos três padrões de reconhecimento conforme Honneth: amor, direito e solidariedade. Materiais e Métodos: Revisão integrativa da literatura, com busca nas bases de dados eletrônicas, nacionais e internacionais, a partir de descritores controlados e palavras relacionadas. Utilizou-se também a obra "Luta por Reconhecimento: a gramática moral dos conflitos sociais". Resultados: Foram incluídos 16 artigos na revisão. Os resultados apontaram a práxis da enfermagem frente à escolha/recusa do cuidado respaldado no respeito mútuo, nas legalidades e nas reflexões frente à complexidade subjetiva do paciente e sua autonomia. Discussão: O amor, direito e solidariedade, compreendidos no processo de cuidado em enfermagem são vistos na literatura como uma construção histórica e social de reconhecimento mútuo, assim como relação intersubjetiva entre o profissional e o indivíduo, buscando estabelecer respeito à dignidade humana e à sua autonomia. Desrespeitar tais relações resultam em desumanização e desmoralização do sujeito. Conclusões: $\mathrm{O}$ respeito às dimensões do amor, direito e solidariedade contribuem para o reconhecimento do cuidado de enfermagem e autonomia do paciente.

Palavras-chave: Cuidados de Enfermagem; Enfermagem; Filosofia; Filosofia em Enfermagem; Hospitais.
Recebido: 13 de abril de 2020

Aceito: 16 de setembro de 2020

Publicado: 19 de fevereiro de 2021 $\square *$ Correspondência

Carolina Huller Farias

E-mail:carolinahuller@gmail.com 


\section{Honneth: Contributions to Nursing Care in Light of Love, Rights and Solidarity}

\section{Abstract}

Introduction: Understanding the intersubjective relationship that permeates nursing care requires mutual recognition between nurses and patients and/or families. Therefore, a reflection on the construction of the human being based on love, rights and solidarity is required. Objective: To reflect on knowledge and practice of nursing care based on respect for the three forms of recognition, love, rights and solidarity, defined by Honneth. Materials and Methods: A comprehensive literature review and search in electronic national and international databases were carried out using controlled descriptors and related terms. The Struggle for Recognition: The Moral Grammar of Social Conflicts was also used for this study. Results: 16 articles were selected to be included in this review. The results addressed nursing practice in the face of choice/ rejection of care based on mutual respect, legalities and reflections on subjective complexity and patient autonomy. Discussion: From a nursing care perspective, love, rights and solidarity are considered in the literature as a historical and social construction mutually recognized as well as an intersubjective relationship between nursing professionals and patients, thus seeking to establish respect for human dignity and autonomy. The lack of respect for such relationships leads to subject dehumanization and demoralization. Conclusion: Respect for love, rights and solidarity dimensions contribute to recognizing nursing care and patient autonomy.

Keywords: Nursing care; Nursing; Philosophy; Philosophy, Nursing; Hospitals.

\section{Honneth: Contribuciones para cuidar en enfermería a la luz del amor, la ley y la solidaridad}

\section{Resumen}

Introducción: La comprensión de la relación intersubjetiva que impregna el cuidado de enfermería requiere un reconocimiento mutuo entre la enfermera y el paciente y / o la familia. Por lo tanto, es necesario reflexionar sobre la construcción del ser humano consolidado en amor, derecho y solidaridad. Objetivo: Reflexionar sobre el conocimiento y la práctica de los cuidados de enfermería basados en el respeto a los tr es estándares de reconocimiento según Honneth: amor, derecho y solidaridad. Materiales y métodos: Revisión integral de literatura, búsqueda en bases de datos electrónicas, nacionales e internacionales, utilizando descriptores controlados y palabras relacionadas. También se utilizó el trabajo "Luta por Reconhecimento: a gramática moral dos conflitos sociais". Resultados: se incluyeron 16 artículos en la revisión. Los resultados apuntaron a la praxis de enfermería frente a la elección / rechazo de la atención basada en el respeto mutuo, las legalidades y las reflexiones sobre la complejidad subjetiva y la autonomía del paciente. Discusión: El amor, el derecho y la solidaridad, entendidos en el proceso de cuidados de enfermería, se ven en la literatura como una construcción histórica y social de reconocimiento mutuo, así como una relación intersubjetiva entre el profesional y el individuo, buscando establecer el respeto por la dignidad humana y su autonomía. La falta de respeto a tales relaciones da como resultado la deshumanización y la desmoralización del sujeto. Conclusiones: El respeto por las dimensiones del amor, el derecho y la solidaridad contribuyen al reconocimiento del cuidado de enfermería y la autonomía del paciente.

Palabras clave: Atención de enfermería; enfermería; Filosofía; Filosofía en Enfermería; Hospitales. 


\section{Introdução}

O saber em enfermagem, seja por métodos científicos ou provindo da sabedoria convencional, foi construído por múltiplas formas de conhecimento, o que contribuiu para a consolidação da enfermagem como profissão'. Embora com reflexo histórico de restritas atividades tecnicistas, atualmente, a práxis da enfermagem requer maiores reflexões, adoção de pensamentos críticos e empoderamento frente às demandaš2. Entretanto, retomando ao conceito de "realização de uma atividade de forma consciente e orientada, que envolve aspectos objetivos e subjetivos $[. . .]^{\prime 2}$, p.2826, a práxis de enfermagem, por vezes, é caracterizada pelo cuidado que nem sempre é entendido como tal.

Para compreender o cuidado enquanto prática laboral é preciso compreender as relações entre as percepções do profissional e às percepções do paciente. Nesse sentido, ilustrando as relações intersubjetivas no cuidar de enfermagem, um estudo, desenvolvido em ambiente hospitalar, identificou concordâncias entre pacientes e enfermeiros em itens considerados mais importantes sobre o ato de cuidar. Foram considerados: o ouvir atentamente; apoiar e ajudar o paciente; monitorar o paciente e; realizar um bom cuidado corporal ${ }^{3}$. O cuidar, portanto, permeia por ações, emoções e sensibilidades com a finalidade de atendimento de todas as demandas da singularidade humana ${ }^{4}$.

Diversas interpretações do cuidar de enfermagem são percebidas sob a ótica dos pacientes, visto suas singularidades e experiências ${ }^{5}$. No entanto, sabe-se que a ocorrência do cuidado se dá a partir da real relação de atribuir e de receber o cuidado, um elo de integração profissionalpaciente. Nessas relações intersubjetivas, as percepções do comportamento de cuidado por parte dos pacientes são inferiores às identificadas pelos profissionais de saúde ${ }^{3,6,7}$. Em outras palavras, nem todos os cuidados de enfermagem ofertados, efetivamente, garantem a sensibilidade dos pacientes em sentir-se sendo cuidado. Além disso, pacientes tendem a uma maior valorização das atividades de cuidados relacionadas ao uso de instrumentais e habilidades técnicas dos profissionais ${ }^{7}$.

Em diversos cenários assistenciais, sobretudo, no contexto hospitalar, a assistência clínica à saúde é acompanhada pela automatização, dada a inserção dos variados aparatos tecnológicos. No que tange ao cuidar de enfermagem - que requer muito além da operacionalização de instrumentos - há afirmativas de que, a falta de domínio de tecnologias, somada à falta de capacitação oferecida pela instituição de saúde, pode gerar insatisfação profissional e poderá resultar em um trabalho desempenhado de forma mecânica, pouco afetiva e autolimitadå ${ }^{8}$.

Nesse sentido, os aparatos tecnológicos apresentam-se como um desafio no cenário de cuidado. Nas unidades de alta complexidades, por inúmeras vezes, aos equipamentos e maquinários são incumbidos a responsabilidade de intermediar a comunicação entre o doente crítico e a equipe $\mathrm{e}^{9-10}$. Portanto, questiona-se se a inserção contínua das tecnologias de produto pode propiciar perdas na relação do cuidar ${ }^{8}$.

$\mathrm{Na}$ desconstrução da ideia automatizada do cuidar, cabe retomar a construção do próprio ser humano, que vai além da constituição material. O ser humano trata-se de uma formação biopsicossocial, dotado de complexidade e subjetividade 4 . Dessa forma, o cuidado atribuído a um corpo qualquer, sem reflexão desse processo histórico, não transcende à pura realização de um procedimento ${ }^{11}$.

A posição de rebaixamento e vulnerabilidade atribuída ao indivíduo hospitalizado, por adoecimento ou demais outras demandas de saúde, retoma a importância do fortalecimento 
do ser humano frente sua experiência de reconhecimento. Tal dependência construtiva do ser em relação à sua identidade dar-se a partir de um reconhecimento recíproco colhido nas relações intersubjetivas ${ }^{12}$.

As relações intersubjetivas fazem o cuidado integral de enfermagem. Nessas relações são consideradas o acolhimento, a responsabilização e a ótica de novas possibilidades, tanto para quem cuida, como também para quem demanda de cuidado. Deste modo, há a transformação dos indivíduos em seres autônomos e criativos, em busca de padrões normativos que respeitem os valores e necessidades biopsicossociais ${ }^{13}$.

Por outro lado, a ruptura dessas relações intersubjetivas representa a forma mais elementar de rebaixamento pessoal. Tem-se a ruptura quando privação dos direitos básicos, maustratos, violação e exclusão, resultando em desrespeito e comprometimento da integridade do indivíduo como um ser, o que implica diretamente sobre o processo de adoecimento ${ }^{12}$.

Em suma, ofertar o cuidado se mostra como a arte de ir além das execuções tecnicistas. Claramente, em situações emergenciais é demonstrada a prioritária importância na condução de atividades tecnicistas, coordenadas e bem desempenhadas ${ }^{14}$. No entanto, na relação cotidiana enfermeiro-paciente, espera-se o extrapolar destas atividades, a partir da relação intersubjetiva de construção mútua entre os elos envolvidos.

De outra forma, mesmo que as atividades tecnicistas sobressaem durante as demandas de cuidado, igualmente é esperado que o profissional assuma uma postura acolhedora. Para tanto, o profissional de enfermagem, dada sua imperativa posição "do saber" não pratique "o fazer" facilitando a ocorrência de situações que expõe o paciente em condições vexatórias, que resultam em insegurança social e comprometimento de sua psiquê ${ }^{12}$. Isto é, na visão ampliada do cuidado de enfermagem, a interação profissional-paciente seja respaldada no respeito às singularidades do outro, nas decisões eticamente fundamentadas e na dedicação emotiva e de confiança.

A disciplina "Autonomia, cuidado em saúde e reabilitação" do Programa de Pós-Graduação em Enfermagem da Universidade Federal de Santa Catarina, foi palco para discussões e reflexões acerca do saber e fazer da enfermagem. Dessa forma, partindo do pressuposto de que o cuidado de enfermagem ofertado com respeito às singularidades humanas reflete positivamente nas relações enfermeiro-paciente, suscitou o seguinte questionamento: Como ofertar o cuidado de enfermagem com respeito ao amor, o direito e a solidariedade ao paciente?

Ao compreender o complexo relacionamento intersubjetivo que envolve o cuidado de enfermagem e o desafio da visão ampliada do cuidar, objetivou-se com este estudo refletir sobre o saber e fazer desse cuidado a partir do respeito aos três padrões de reconhecimento conforme Honneth: amor, direito e solidariedade. 


\section{Materiais e métodos}

Trata-se de uma revisão integrativa da literatura, desenvolvida no período de junho a julho de 2019. Foi escolhido esta proposta metodológica por propiciar a discussão de um assunto a partir do ponto de vista teórico ou contextual, ancorado por estudos relevantes que dão suporte para a melhoria da prática clínica ${ }^{15}$. Tais análises permitem, portanto, uma reflexão teórica da literatura a partir das interpretações críticas dos autores refletidas ainda, pelas discussões realizadas em sala de aula e suas vivências em ambientes de cuidado.

O corpus da pesquisa foi selecionado a partir de buscas nas bases de dados eletrônicas: Biblioteca Virtual da Saúde (BVS); Scientific Electronic Library Online (SciELO); SCOPUS, Academic Search Premier, SocINDEX e Cumulative Index to Nursing Allied Health Literature (CINAHL). Utilizou-se os descritores indexados: Enfermagem; Cuidados de Enfermagem; Humanização da Assistência; Amor; Solidariedade; Direitos Civis; Filosofia; Filosofia em Enfermagem; associados à sinônimos, em inglês, português e espanhol. Realizou-se o cruzamento pelos operadores booleanos AND e $O R$.

Foram adotados os seguintes critérios para seleção dos estudos: artigos originais, completos e disponíveis online; nos idiomas inglês, português ou espanhol; publicados de 2015 a 2019; e que respondessem ao objetivo proposto pelo estudo. Foram excluídos: resumos publicados em anais de eventos científicos, relatórios governamentais, leis, decretos, teses e dissertações, revisões da literatura e artigos publicados em revistas não indexadas, estudos fora do contexto profissional e duplicados.

Títulos e/ou resumos das referências identificadas nas bases de dados foram lidos, e, após aplicado os critérios de elegibilidade, foram pré-selecionados aqueles elegíveis para o estudo. Dessa forma, um pesquisador leu, de forma criteriosa artigos completos pré-selecionados. Posteriormente, um segundo pesquisador confirmou a seleção dos materiais que contemplassem a temática para compor o corpus de análise.

A fim de assegurar o desdobramento da discussão reflexiva na perspectiva dos três padrões de reconhecimento segundo Axel Honneth utilizou-se a sua obra traduzida intitulada: Luta por Reconhecimento: a gramática moral dos conflitos sociais ${ }^{12}$.

O relato deste estudo de revisão se deu de forma narrativa, a partir das discussões suscitadas pelos artigos selecionados para sustentação teórica e a obra de referência previamente mencionada. Além disso, a seleção e identificação dos estudos foi representado em fluxograma de acordo com Preferred Reporting Items for Systematic Reviews And Meta-Analyses (PRISMA) ${ }^{16}$. Ademais, os resultados principais foram apresentados em um quadro, em que os estudos foram representados com a letra e número E1, E2, E3..., sucessivamente na ordem de apresentação.

\section{Resultados}

Na busca inicial nas bases de dados encontrou-se 1.257 publicações. Após leitura de títulos e/ou resumos, e aplicando os critérios de elegibilidade, foram pré-selecionados 34 artigos, os quais, após leitura na íntegra pelos pesquisadores, 16 resultaram para a sustentação teórica (Figura 1). 


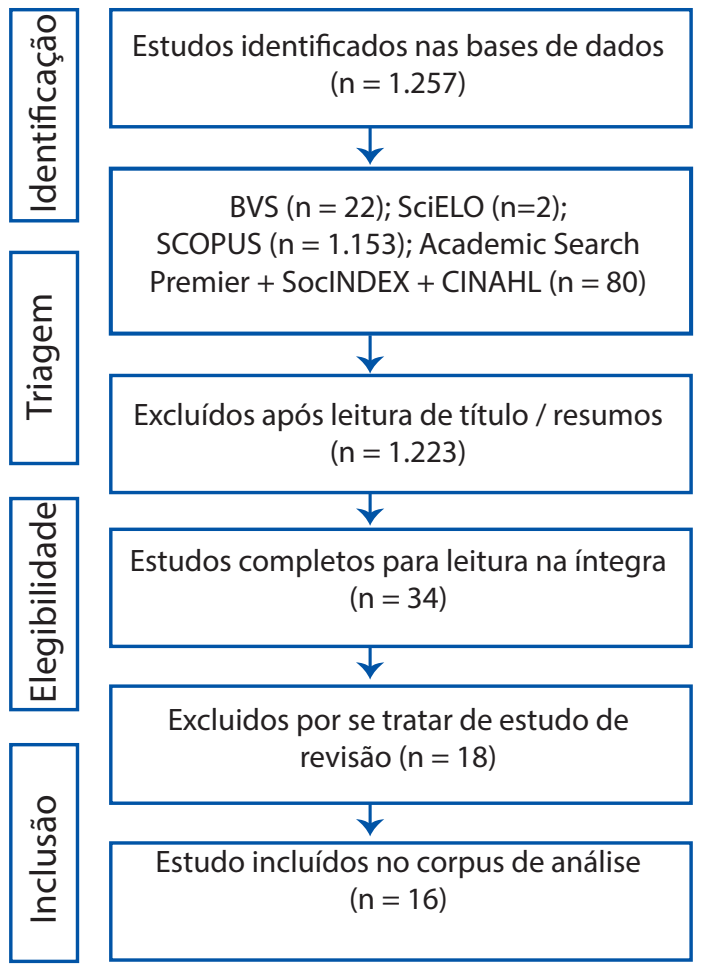

Figura 1 - Percurso para busca e seleção dos artigos nas bases de dados, Florianópolis, SC, Brasil, 2020.

Dos 16 artigos incluídos na revisão integrativa (Quadro 1) cinco eram estudos desenvolvidos no Brasil, e os demais, internacionais. Todos os artigos selecionados foram publicados entre 2015 e 2018 com maior concentração no ano de 2016, com seis estudos publicados. Foram selecionados estudos com delineamento quantitativo e qualitativo.

Quadro 1 - Apresentação dos artigos selecionados, de acordo país, ano, método e resultados principais ( $n=16)$, Florianópolis, SC, Brasil, 2020.

\begin{tabular}{|c|c|c|}
\hline Estudo & $\begin{array}{l}\text { País/ Ano/ } \\
\text { Método }\end{array}$ & Resultados principais e conclusões \\
\hline$E 1^{14}$ & $\begin{array}{c}\text { Brasil/ 2016/ } \\
\text { Estudo Descritivo }\end{array}$ & $\begin{array}{l}\text { Os resultados mostraram a percepção dos sentimentos gerados no } \\
\text { momento da recusa do cuidado pelo paciente em situação de } \\
\text { emergência e quais sentidos os profissionais de enfermagem dão a } \\
\text { esse ato. Alguns se sentiram desvalorizados, constrangidos, } \\
\text { indiferentes ou até mesmo com raiva. Outros demonstraram que a } \\
\text { recusa pode se dar por diversos fatores, como a falta de } \\
\text { conhecimento sobre sua atual situação clínica, por medo ou por falta } \\
\text { de uma participação efetiva da equipe multiprofissional. }\end{array}$ \\
\hline$E 2^{17}$ & $\begin{array}{c}\text { Estados Unidos/ 2016/ } \\
\text { Estudo Etnográfico }\end{array}$ & $\begin{array}{l}\text { Os enfermeiros descreveram quatro tipos de habilidades - } \\
\text { observacional, analítica, interacional e reconfortante - que eles } \\
\text { empregam na realização do trabalho/cuidado de enfermagem à beira } \\
\text { do leito. }\end{array}$ \\
\hline $\mathrm{E}^{18}$ & $\begin{array}{l}\text { Brasil/ 2017/ Estudo } \\
\text { Fenomenológico }\end{array}$ & $\begin{array}{l}\text { Familiares acompanhantes vivenciam sofrimentos ambíguos, } \\
\text { classificados em três dimensões: coexistência entre familiar } \\
\text { acompanhante e idoso hospitalizado, o dever legal e moral no } \\
\text { cuidado ao idoso hospitalizado, e o des(cuido) no serviço hospitalar. }\end{array}$ \\
\hline
\end{tabular}




$\begin{array}{lll}\text { Estudo } & \text { País/ Ano/ } & \text { Mesultados principais e conclusões }\end{array}$

E4 ${ }^{19} \quad \begin{gathered}\text { Brasil/ } 2017 / \\ \text { Estudo Descritivo }\end{gathered}$

$E 5^{20}$

Brasil/ 2017/

Estudo Descritivo

$E^{21}$

$\mathrm{E}^{22}$

Canadá/ 2018/

Estudo de Caso

$\mathrm{E}^{23}$

Nova Zelândia/ 2016/

Estudo Fenomenológico

E924

China/ 2016/

Estudo Transversal

$\mathrm{E} 10^{25}$

Chile/ 2018/

Estudo Transversal

E11 16

Canadá/ 2015/

Estudo Descritivo

E12 27
Brasil/ 2016/

Estudo Descritivo
A simpatia foi reconhecida como elemento fundamental para o cuidado de enfermagem ao paciente crítico na Unidade de Terapia Intensiva. A simpatia foi manifestada na práxis assistencial a partir de atitudes, tais como 'chegar perto', 'ter proximidade', 'oferecer uma palavra de carinho e conforto', 'conversar com o paciente', 'acolher aquele que sofre'.

A utilização da música é uma das maneiras de promover a saúde das crianças a fim de proporcionar conforto, comunicação e melhorar a relação profissional de saúde com o paciente tornando o cuidado mais humanizado.

No contexto da prática clínica e da pesquisa, o respeito à autonomia está intimamente ligado ao valor da privacidade, na liberdade da tomada de decisões orientada por uma ética de cuidado e responsabilidade moral.

Os enfermeiros gerenciam as tensões inerentes a assistência de enfermagem, e a enfermagem hábil pode ter o efeito de tornar essas tensões invisíveis. Isso coloca os enfermeiros em uma posição eticamente complexa, onde, sua empatia humanizadora tem o potencial de melhorar a assistência e o resultado do cuidado.

A hospitalidade se mostrou de maneiras diferentes para os participantes. Quando presentes, eles experimentaram sentimentos de conforto, de estar à vontade e de se sentir melhor. As implicações para os profissionais de saúde são que a oferta de pequenos atos de hospitalidade pode evocar experiências poderosas que beneficiam o paciente, sugerindo que o cuidado com o paciente é um elemento importante para o processo de cura.

Enfermeiros que aceitam ajuda, apoio e carinho de seus colegas, desenvolvem um melhor desempenho no trabalho e consequentemente, têm o seu humor melhorado, refletindo positivamente na assistência oferecida ao paciente.

Pacientes hospitalizados percebem o cuidado de forma humanizada. A maneira de realizar os cuidados é através do desenvolvimento de um vínculo enfermeiro-paciente. Esse vínculo é obtido considerando as características do profissional, habilidades técnicas, conhecimentos e atitudes como empatia, respeito e autenticidade.

A justiça social foi descrita pelos enfermeiros como base para a práxis de enfermagem. Os enfermeiros testemunham rotineiramente os impactos da injustiça social na vida de seus clientes/pacientes, e acabam desempenhando fortemente um papel de 'advocacia', ou seja, são guiados por um princípio cujo imperativo moral está embasado no apoio as ações sociais de forma coletiva, ações essas que promovem a justiça social e a equidade em saúde na sociedade moderna.

Os enfermeiros reconhecem o valor do cuidado espiritual e estão cientes de que as necessidades espirituais são muitas vezes colocadas em segundo plano para dar lugar ao tratamento físico. Os enfermeiros justificaram a ausência de cuidado espiritual para as crianças embasados na falta de tempo, na idade e nível de consciência/maturidade das crianças. 


\begin{tabular}{|c|c|c|}
\hline Estudo & $\begin{array}{l}\text { País/ Ano/ } \\
\text { Método }\end{array}$ & Resultados principais e conclusões \\
\hline $\mathrm{E}^{2} 3^{28}$ & $\begin{array}{l}\text { Canadá/ 2015/ } \\
\text { Estudo Descritivo }\end{array}$ & $\begin{array}{l}\text { A simpatia foi descrita como uma resposta indesejada e frente a uma } \\
\text { situação angustiante, caracterizada pela falta de compreensão e } \\
\text { autopreservação do observador. A empatia foi vivida como uma } \\
\text { resposta afetiva que reconhece e tenta entender o sofrimento do } \\
\text { indivíduo. A compaixão aprimorou as principais facetas da empatia ao } \\
\text { adicionar características distintas de ser motivado pelo amor, da ação } \\
\text { e de pequenos atos de bondade. Os pacientes relataram que, } \\
\text { diferentemente da simpatia, a empatia e compaixão eram benéficos, } \\
\text { sendo a compaixão a mais preferida e impactante. }\end{array}$ \\
\hline $\mathrm{E} 14^{29}$ & $\begin{array}{c}\text { Noruega/ 2015/ } \\
\text { Pesquisa Hermenêutica }\end{array}$ & $\begin{array}{l}\text { Os resultados revelaram o núcleo do amor em quatro dimensões: o } \\
\text { amor como força motriz interior, buscando o ser humano em sua } \\
\text { essência, a fé compreendida como uma força interior para alcançar a } \\
\text { recuperação em saúde e o amor como um movimento de dar e } \\
\text { receber. A interpretação hermenêutica revelou o núcleo do amor } \\
\text { como sacrifício, mostrando que o sacrifício é uma dimensão ética e } \\
\text { que envolve a busca ontológica do sofrimento no paciente. O } \\
\text { sacrifício está conectado à fé, e a fé no amor é decisiva para alcançar a } \\
\text { cura/reabilitação. }\end{array}$ \\
\hline $\mathrm{E} 15^{30}$ & $\begin{array}{l}\text { Colômbia/ 2016/ } \\
\text { Estudo Fenomenológico }\end{array}$ & $\begin{array}{l}\text { O cuidado humanizado requer o envolvimento de profissionais, } \\
\text { instituição e paciente, com o objetivo de recuperação em saúde. } \\
\text { Ofertar o cuidado humanizado em prática requer a soma dos esforços } \\
\text { de instituições e enfermeiros dentro de uma estrutura que apoia as } \\
\text { propostas de implementar planos de cuidados e cursos de ação } \\
\text { baseados em abordagens teóricas e éticas que favorecem a gestão e } \\
\text { liderança de enfermagem. Isso é mais compatível com uma proposta } \\
\text { de assistência humanizada do que aqueles que sacrificam o } \\
\text { bem-estar dos enfermeiros e pacientes e limitam os recursos e } \\
\text { serviços com o único propósito de lucratividade. }\end{array}$ \\
\hline$E 16^{31}$ & $\begin{array}{l}\text { Portugal/ 2018/ } \\
\text { Estudo Transversal }\end{array}$ & $\begin{array}{l}\text { A compreensão das dimensões da vulnerabilidade, assim como sua } \\
\text { experiência, permite ao profissional entender melhor o paciente, } \\
\text { facilita o desenvolvimento da empatia e desperta os enfermeiros para } \\
\text { a necessidade de melhorar a qualidade do atendimento. A } \\
\text { vulnerabilidade pode ser um aliado para que o profissional dê mais } \\
\text { atenção ao paciente, para atribuir-lhe maior importância e envolvê-lo } \\
\text { no planejamento do cuidado, estimulando o paciente a formação de } \\
\text { sua própria autonomia. }\end{array}$ \\
\hline
\end{tabular}

\section{Discussão}

O cuidado de enfermagem é de suma importância para restabelecimento das condições clínicas do indivíduo. O cuidado, enquanto atividade profissional, excede a realização de tarefas, tratase da aplicação de um conhecimento histórico e cientificamente fundamentado, justificado por reflexões e análises teóricas ${ }^{17,25}$. No entanto, a enfermagem, e, particularmente, o cuidado, não é percebido como uma ferramenta de trabalho pelos pacientes, ainda que historicamente consolidado como tal. Dessa forma, argumenta-se da importância do reconhecimento do paciente quanto os cuidados a ele oferecidos ${ }^{14}$.

Anteriormente ao reconhecimento do cuidado pelo paciente, deve-se haver a concordância para recebê-lo. O cuidado, como atividade da rotina assistencial de enfermagem, parte da interação entre dois ou mais envolvidos, que ocorre de forma consentida ${ }^{17,29}$. No entanto, contrariamente, 
são observadas a não concessão na recepção de cuidados. A frequência maior de recusa, pelos pacientes, ocorre durante internação em unidades de alta complexidade, especialmente, unidades de emergências ${ }^{14}$. De maneira geral, nessas unidades são entregues aos pacientes, um diagnóstico médico, previamente desconhecido e que provoca não aceitabilidade ${ }^{14}$; além disso, a característica de unidades com período curto de internação contribui para relação de confiança fragilizada do paciente ao profissional ${ }^{17}$.

Ademais, o próprio cenário ritualístico e tecnicista, sobretudo nas situações de institucionalização, corrobora a pressão sobre as escolhas do paciente e/ou familiar. Entretanto, cabe ao paciente a liberdade da recusa de intervenções assistenciais, conquanto usufrua da plenitude de suas faculdades mentais e não esteja em iminência de morte ${ }^{14}$.

Assim, o envolvimento por parte do paciente, quanto a aceitabilidade de todas as ações a ele impostas no transcorrer da internação hospitalar, é incerto ${ }^{17}$. Por outro lado, contrapõe a certeza de que o trabalho profissional deva ser executado, em data e hora marcada. Nesse sentido, a interação conexa e respeitosa entre enfermeiro-paciente contribui para que as melhores escolhas sejam tomadas ${ }^{29}$.

A fim de dissipar dilemas éticos que perpassam no âmbito de relacionamento social enfermeiropaciente ${ }^{22}$, cabe o reconhecimento mútuo dos direitos e dos deveres entre os elos envolvidos ${ }^{14}$. Dessa forma, cria-se uma base para a busca conjunta de melhores formas de enfrentamento ${ }^{12}$. Como consequência, laços de cumplicidade e respeito são estabelecidos.

Em contrapartida, é desafiador o cenário do enfermeiro frente ao paciente que recusa cuidados ou não reconhece a necessidade dos cuidados a ele oferecidos. No entanto, como profissional educador em saúde, cabe o esclarecimento da importância dos cuidados, possíveis consequências e, especialmente, os benefícios na aceitabilidade, alegando almejar o bem humano, que é um compromisso ético e legal do profissional ${ }^{22}$. De maneira geral, as orientações advindas do profissional sobressaem, quando praticadas na relação de respeito ao paciente e suas decisões ${ }^{21}$. Dessa forma, se promove o respeito à dignidade humana e à sua autonomia ${ }^{14,21}$.

De acordo com Honneth, a conquista da autorrealização pessoal se desdobra frente a uma construção histórica e social de reconhecimento de forma contínua. Para isso, são determinadas três dimensões essenciais: o amor, o direito e a solidariedade, dos quais, quando efetivamente praticados e percebidos resultam em autoconfiança, autorrespeito e autoestima, respectivamente. Enquanto cuidado de enfermagem, tais dimensões retomam como uma prática emancipadora.

Paradoxalmente, a experiência de desrespeito em qualquer das três dimensões (amor, direito e solidariedade) reflete de duas maneiras no indivíduo. Se de um lado impacta na dignidade humana, limitando o sujeito frente sua realidade moral. Por outro lado, tem o potencial de promover reflexões com vistas à busca pela valoração da autorrealização, ou seja, em busca da emancipação em resistência às forças imperativas normativas de desrespeito ${ }^{12}$.

Na enfermagem, é a partir do amor e empatia que o vínculo é estabelecido. A adoção de condutas praticadas a partir destes, permite o conhecimento da própria essência, ou seja, autoconfiança, e proporciona uma comunicação clara entre enfermeiro-paciente ${ }^{19,28}$. Guiado nesta relação, 0 
enfermeiro consegue estabelecer o cuidado rotineiro que o torna qualificado para conhecer e atender à necessidade do próximo, e afasta a aplicação de terapêuticas baseadas somente em aspectos biológicos que não consideram o homem em sua completude ${ }^{29}$.

Aproximando os padrões de reconhecimento de Honneth à enfermagem, o amor significa dar e providenciar um cuidado com o objetivo de restaurar o equilíbrio físico-emocional. Neste aspecto, a conexão com o paciente torna-se fundamental para compreender a real dimensão do sofrimento ${ }^{29}$, de forma a direcionar positivamente seu trabalho ${ }^{24}$.

Por outro lado, o cuidar em enfermagem torna-se prejudicado quando há a transgressão dos valores éticos, resultando em desumanização e desmoralização do sujeito. O enfermeiro, quando deixa de agir com humanidade na situação de cuidado, expõem-se à estagnação do seu crescimento e desenvolvimento sustentado no amor, visto como elemento essencial para a práxis assistencial da enfermagem ${ }^{19}$. Dessa forma, tem-se o rompimento com o compromisso de promover a autoconfiança.

O desrespeito ao direito e solidariedade, no entanto, podem extrapolar a capacidade profissional do enfermeiro. Sentimentos de medo, insegurança e saudades do ambiente familiar avassalam pacientes e familiares, sobretudo, quando, no período de internação, ficam alojados nos estreitos corredores, sem privacidade ou quaisquer confortos ${ }^{18}$. Entretanto, enquanto gestão do cuidado, enfermeiros podem não apenas apontar os problemas vivenciados em suas unidades de trabalho aos dirigentes dos serviços de saúde, como também, atuar frente à defesa dos direitos dos pacientes.

Situações precárias, tanto de recursos materiais quanto recursos humanos, identificadas no cotidiano dentro das instituições de saúde, demonstram o quanto a organização dos serviços podem refletir diretamente no cuidado de enfermagem ${ }^{31}$. No entanto, os profissionais têm utilizados estratégias que buscam uma resposta positiva do cuidar, tais como resgatar na memória um ambiente familiar ao paciente, e amenizar o sofrimento mental, que pode ser provocado pela institucionalização $20,23,24$.

No contexto hospitalar, o cuidado como forma intersubjetiva de se relacionar, inicia-se no momento do acolhimento, saída do mundo real, rotineiro, familiar, para embarcar em uma realidade normativa institucional. O inesperado diagnóstico médico que, por consequência impõe a necessidade da internação hospitalar, apresenta-se em muitos cenários como um processo difícil e traumático ${ }^{18}$. Eliminá-lo, portanto, vislumbra-se utópico, mas fornecer uma ambiência ${ }^{20,23}$, com atribuições compartilhadas, visando a construção multiprofissional do cuidar ${ }^{14}$ são artifícios para amenizar seu impacto negativo.

De outra maneira, a própria monotonia da rotina da enfermagem contribui para o desrespeito profissional quanto ao direito e a solidariedade. Na área da saúde, foram integrados aparatos tecnológicos, com o advento da era industrial ${ }^{21}$; no cenário de cuidado, particularmente em ambientes hospitalares, a enfermagem trabalha em acordo com aparelhos e máquinas, desafiando, sobremaneira, os profissionais, dada a possibilidade de deixar de ouvir o paciente por estar ensurdecido pelos ruídos produzidos por essas tecnologias, gerando estresse e indiferença ao paciente ${ }^{14}$.

Estudo conduzido com profissionais da saúde sobre a dimensão da vulnerabilidade, demonstra que a vulnerabilidade é algo indissociável do ser humano, sendo, portanto, uma realidade 
inseparável do fator "existência". Nos resultados apresentados, observa-se a exclusão da participação dos pacientes no planejamento do próprio cuidado, pelos profissionais, adotando um modelo paternalista ${ }^{31}$. Deste modo, verifica-se a vulnerabilidade dos pacientes, ao se negar autonomia na escolha da sua terapêutica, portanto, uma forma de exclusão social.

A exclusão social é caracterizada pela falta de reconhecimento, desrespeito e desvalorização do indivíduo em sua intersubjetividade ${ }^{26}$. Envolve rejeitar aqueles que diferem dos padrões da sociedade bem como subordiná-los, negando o reconhecimento de sua identidade e autonomia ${ }^{12,26}$.

Para isso, reconhecer o indivíduo, não como seu objeto de trabalho, mas como uma construção biopsicossocial, regrado de moralidades, recoberto de anseios, desejos, angústias, percepções, enfim, um ser complexo, subjetivo e singular, torna-se um desafio diário para a práxis da enfermagem, o que retoma a necessidade reflexiva do cuidar. A compreensão do visível e do invisível, do corpo adoecido como total atenção profissional, que quando descuidado, reflete a invisibilidade da atenção, o mesmo ao familiar ao lado, coexistindo no sofrimento do ente, confuso na sua dupla posição, entre a "obrigação" do cuidar e a subordinação institucional ${ }^{25}$.

Nessa troca, o enfermeiro enquanto gestor e prestador do cuidado observa o indivíduo e todas as suas formas de comunicação, verbalmente ou não, na busca incessante de identificar suas reais demandas, principalmente às que em suma passam despercebidas, como as demandas espirituais ${ }^{27}$.

Cada pessoa tem sua forma de se comunicar ${ }^{18,31}$, e também a liberdade de ficar calada ${ }^{17}$, logo a escuta qualificada torna-se uma leal aliada para essa percepção necessária ${ }^{17,28,29}$, que auxilia na elaboração do cuidado personalizado27,29.

Neste sentido, e em uso da lente de reconhecimento honnethiana, faz-se necessário o exercício de identificar as formas de desrespeitos institucionais, os conflitos intersubjetivos, as relações profissionais ${ }^{24}$, as demandas de saúde em estágio irresolutas e as vulnerabilidades ${ }^{31}$. Portanto, identificar os atributos imersos em toda pluralidade do cuidar como ato relacional, para a busca conjunta, planejada, dialogada da melhor e mais efetiva maneira de cuidar, ou seja, aquela em que os envolvidos percebam e se beneficiem com tal ${ }^{12,19,31}$.

De tal forma, o exercício crítico e reflexivo contribui sobremaneira para o enfermeiro e sua rotina de trabalho. Dado que, resgatar os atributos imersos na pluralidade do cuidar enquanto relação intersubjetiva promove a oportunidade profissional de melhoria contínua do cuidado, frente ao aprendizado concebido ${ }^{31}$, fortalecendo assim, sua autonomia, bem como respeitando a autonomia e dignidade do paciente ${ }^{14}$. E desta maneira, contribui ao profissional para continuamente se apropriar da prática em ofertar um cuidado ampliado de enfermagem. Por conseguinte, desconstrói-se um entendimento, comum e cômodo no contexto hospitalar, das atividades de cuidado pautadas em procedimentos, seguidas na lógica fragmentada do ser e que desvaloriza a prática reflexivo-filosófica do cuidar de enfermagem.

Resgatar os atributos imersos na pluralidade do cuidar enquanto relação intersubjetiva promove a oportunidade profissional de melhoria contínua do cuidado, frente ao aprendizado concebido ${ }^{31}$, fortalecendo assim, sua autonomia, bem como respeitando a autonomia e dignidade do paciente ${ }^{14}$ 


\section{Conclusões}

Os desafios para uma visão ampliada do cuidado corroboram o crescimento mútuo enfermeiropaciente, quando, então, o cuidado, visto na sua relação intersubjetiva, é ofertado a partir do respeito recíproco, nas legalidades da profissão e civis, e nas reflexões frente à complexidade subjetiva do paciente e sua autonomia.

A compreensão do cuidado é alcançada, a partir do estabelecimento de vínculos construídos pelo conhecimento profissional, pelas relações afetivas, clara comunicação, respeito das singularidades, escuta qualificada, compreensão, olhar sensível e contextual, responsabilidade e contínuo desenvolvimento de habilidades enfermeiro-paciente.

Este estudo possui algumas limitações. Restrições aplicadas quanto ao período de recuperação dos estudos, bem como, quanto ao idioma, impactou no quantitativo final de estudos no corpus de análise. Diante disso, a discussão tornou-se mais restrita aos ambientes de institucionalização, particularmente, unidades hospitalares. No entanto, a enfermagem é praticada em todos os campos onde há necessidade de cuidado de enfermagem, como ambulatórios, residências, escolas, comunidades, consultórios, entre outros.

Por outro lado, os resultados apresentados neste estudo contribuíram para dialogar sobre alguns dos principais conflitos que o enfermeiro precisa manejar na situação de cuidado ao paciente. Além disso, foram apresentadas possibilidades para melhoria da gestão do cuidado, como uma prática emancipatória, com respeito às três dimensões de reconhecimento definidas por Honneth. Desta forma, as discussões suscitadas podem contribuir para enfermeiros, enquanto gestores e prestadores de cuidados; além disso, podem contribuir para dirigentes dos serviços de saúde, vistos argumentações de condições institucionais necessárias para prestação de serviços assistenciais pautadas na dignidade humana e profissional.

\section{Referências}

1. MecEwen M. Filosofia, ciência e enfermagem. In: McEwen M, Wills EM. Bases teóricas de enfermagem. Porto Alegre: Artmed; 2016.

2. Salbego C, Nietsche EA, Teixeira E, Girardon-Perlini NMO, Wild CF, Ilha S. Tecnologias cuidativo-educacionais: um conceito emergente da práxis de enfermeiros em contexto hospitalar. Rev. Bras. Enferm. 2018; 71(6): 2825-33. http://dx.doi.org/10.1590/0034-7167-2017-0753

3. Flynn S. Who cares? A critical discussion of the value of caring from a patient and healthcare professional perspective. Int. J. Orthop. Trauma Nurs. 2016; 20: 28-39. https://doi.org/10.1016/j.ijotn.2015.06.001

4. Monteiro PV, Almeira ANS, Pereira MLD, Freitas MC, Guedes MVC, Silva LF. Quando cuidar do corpo não é suficiente: a dimensão emocional do cuidado de enfermagem. Rev. Min. Enferm. 2018; 20: 1-5. http://www.dx.doi.org/10.5935/1415-2762.20160026

5. Compton E, Gildemeyer K, Mason TM, Hartranft SR, Sutton SK. Nurses' caring behaviors: the perception of patients with cancer at the time of discharge after surgery. Clin. J. Oncol. Nurs. 2018; 22(2): 169-174. http://www.dx.doi.org/10.1188/18.CJON.169-174 
6. Kiliç M, Öztunç G. Comparison of nursing care perceptions between patients who had surgical operation and nurses who provided care to those patients. Int. J. Caring Sci. 2015; 8(3): 625-33.

https://www.internationaljournalofcaringsciences.org/docs/13_Kilic_original_8_3.pdf

7. Papastavrou E, Efstathiou G, Charalambous A. Nurses' and patients' perceptions of caring behaviours: quantitative systematic review of comparative studies. J. Adv. Nurs. 2011; 67(6): 1191-1205. https://doi.org/10.1111/j.1365-2648.2010.05580.x

8. Lima AA, Jesus DS, Silva TL. Densidade tecnológica e o cuidado humanizado em enfermagem: a realidade de dois serviços de saúde. Physis. 2018; 28(3): 1-15. http://dx.doi.org/10.1590/S0103-73312018280320

9. Moreira APA, Escudeiro CL, Christovam BP, Silvino ZR, Carvalho MF, Silva RCL. Uso de tecnologias na terapia intravenosa: contribuições para uma prática mais segura. Rev. Bras. Enferm. 2017; 70(3): 595-601. http://dx.doi.org/10.1590/0034-7167-2016-0216

10.Tavares MMM, Coelho PTG, Lopes TMR. Percepção da família sobre a assistência de enfermagem em uma unidade de terapia intensiva adulta. Rev. Enferm. UFPI. 2018; 8(1): 1722. https://doi.org/10.26694/2238-7234.8117-22

11.Waldow VR. Enfermagem: a prática do cuidado sob o ponto de vista filosófico. Investig. Enferm. Imagen Desarro. 2015; 17(1): 13-25. https://doi.org/10.11144/Javeriana.IE17-1.epdc

12.Honneth, A. Luta por reconhecimento: a gramática moral dos conflitos sociais. Tradução: Luiz Repa - São Paulo: Ed. 34, 2003. 296p.

13.Wernet M, Mello DF, Ayres JRCM. Reconhecimento em Axel Honneth: Contribuições à pesquisa em saúde. Texto Contexto Enferm. 2017; 26(4): 1-8.

https://doi.org/10.1590/0104-070720170000550017

14.Abreu LTA, Berardinelli LMM, Santos MLSC. A recusa do cuidado por paciente em situação de emergência: vivências de profissionais de enfermagem. Rev. Enferm. UERJ. 2016; 24(4): 1-6. https://doi.org/10.12957/reuerj.2016.26000

15. Mendes KDS, Silveira RCCP, Galvão CM. Revisão integrativa da literatura: método de pesquisa para a incorporação de evidências na saúde e na enfermagem. Texto Contexto Enferm. 2008; 17(4): 758-64. https://doi.org/10.1590/S0104-07072008000400018

16.Moher D, Liberati A, Tetzlaff J, Altman DG, The PRISMA Group. Preferred reporting items for systematic reviews and meta-analyses: the PRISMA statement. Plos Medicne. 2009; 6(7): 1-6. https://doi.org/10.1371/journal.pmed.1000097

17.Apesoa-Varano EC. Not Merely TLC: Nurses' Caring Revisited. Qual. Sociol. 2016; 39(1): 2747. https://doi.org/10.1007/s11133-015-9322-3

18. Reis CC, Menezes TMO, Sena ELS. Vivências de familiares no cuidado à pessoa idosa hospitalizada: do visível ao invisível. Saúde Soc. 2017; 26(3): 702-11. https://doi.org/10.1590/S0104-12902017156439

19.Guimarães GL, Matos SS, Ferraz AF, Manzo BF, Sharry S, Souza MAF. Redescoberta da simpatia na prática do enfermeiro em terapia intensiva. Rev. Enferm. UFPE. 2017; 11(2): 491497. https://doi.org/10.5205/reuol.10263-91568-1-RV.1102201702

20.Silva KG, Taets GGC, Bergold LB. A utilização da música em uma unidade pediátrica: contribuindo para a humanização hospitalar. Rev. Enferm. UERJ. 2017; 25(1): 1-5. https://doi.org/10.12957/reuerj.2017.26265

21.Dove ES, Kelly S, Lucivero D, Machirori M, Dheensa S, Prainsack B. Beyond individualism: Is there a place for relational autonomy in clinical practice and research? Clin. Ethics. 2017; 12(3): 150-165. https://doi.org/10.1177/1477750917704156

22.Jager F, Perron A. Caring as coercion: exploring the nurse's role in mandated treatment. $J$. Forensic Nurs. 2018; 14(3): 148-153. https://doi.org/10.1097/JFN.0000000000000207

23. Kelly R, Losekoot E, Wright-Stclair VA. Hospitality in hospitals: The importance of caring about the patient. Hospitality \& Society. 2016; 6(2): 113-29.

https://doi.org/10.1386/hosp.6.2.113_1 
24.Chu LC. Mediating positive moods: the impact of experiencing compassion at work. J. Nurs. Manag. 2016; 24(1): 59-69. https://doi.org/10.1111/jonm.12272

25.Monje PV, Miranda PC, Oyarzún JG, Seguel FP, Flores EG. Percepción de cuidado humanizado de enfermería desde la perspectiva de usuarios hospitalizados. Cienc. Enferm. 2018; 24(5): 1-10. https://doi.org/10.4067/s0717-95532018000100205

26.Yanicki SM, Kushner K, Reutter L. Social inclusion/exclusion as matters of social (in)justice: a call for nursing action. Nurs. Inq. 2015; 22(2): 121-33. https://doi.org/10.1111/nin.12076

27. Nascimento LC, Alvarenga WA, Caldeira S, Mica TM, Oliveira FCS, Pan R, Santos TFM, Carvalho EC, Vieira M. Spiritual Care: The Nurses' Experiences in the Pediatric Intensive Care Unit. J. Religions. 2016; 7(3): 1-11. https://doi.org/10.3390/rel7030027

28.Sinclair S, Beamer K, Hack TF, McClement S, Bouchal SR, Chochinov HM, Hagen NA. Sympathy, empathy, and compassion: A grounded theory study of palliative care patients' understandings, experiences, and preferences. Palliat Med. 2016; 31(5): 437-447.

https://doi.org/10.1177/0269216316663499

29.Thorkildsen KM, Eriksson K, Raholm MM. The core of love when caring for patients suffering from addiction. Scand. J. Caring Sci. 2015; 29(2): 353-60. https://doi.org/10.1111/scs.12171

30.Salazar OAB. The meaning of humanized nursing care for those participating in it: Importance of efforts of nurses and healthcare institutions. Invest. Educ. Enferm. 2016; 34(1): 18-28. http://dx.doi.org/10.17533/udea.iee.v34n1a03

31.Almeida CMT, Rodrigues VM. The relationship between human vulnerability and health care: a study with professionals and students in the health area. Mundo da Saúde. 2018; 42(4): 992-1011. http://doi.org/10.15343/0104-7809.201842049921011 